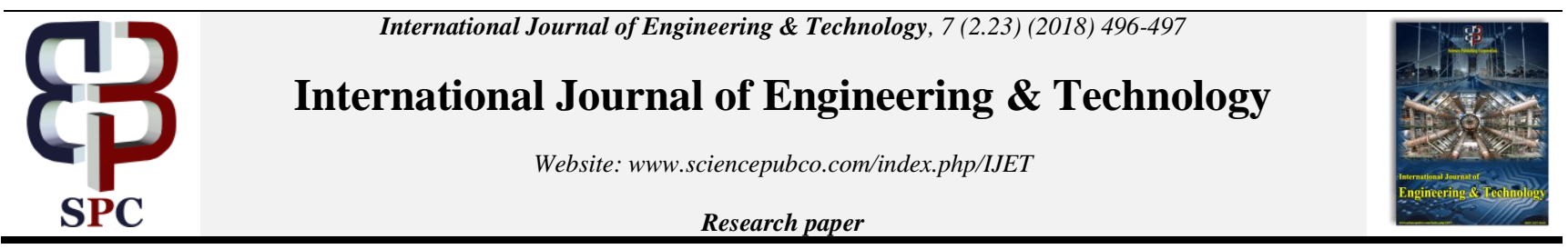

\title{
Calcium Phosphate Bioceramic Material Local Influence on the Bone Biomechanical Properties at Rabbits with Experimental Osteoporosis
}

\author{
Vladislavs Ananjevs ${ }^{1 *}$, Aleksandra Ananjjeva ${ }^{2}$, Janis Vetra ${ }^{3}$, Andrejs Skagers ${ }^{1}$, Ilze Salma ${ }^{1}$, Janis Locs ${ }^{4}$, Vladimir \\ Kasyanov $^{5}$ \\ ${ }^{I}$ Department Of Oral And Maxillofacial Surgery, Riga Stradins University, Riga, Latvia \\ ${ }^{2}$ Children's Clinical University Hospital, Riga, Latvia \\ ${ }^{3}$ Institute Of Anatomy And Athropology, Riga Stradins University, Riga, Latvia \\ ${ }^{4}$ Rudolfs Cimdins Riga Biocomponents Innovations And Development Centre, Institute Of General Chemical Engineering, Faculty Of \\ Component Science And Applied Chemistry, Riga Technical University, Riga, Latvia \\ ${ }^{5}$ Laboratory Of Biomechanics, Riga Stradins University, Riga, Latvia \\ *Corresponding Author E-Mail: V.Ananjevs@Gmail.Com
}

\begin{abstract}
Biomechanical characteristics of the rabbit cartilage component from the femur were investigated. The control osteoporosis had been activated by ovariectomy alongside the injections of methylprednisolone. During the experiment defects in the femur's greater trochanter zone was created and afterwards filled with the fragments of a hydroxyapatite as well as tricalcium phosphate (HAP/TCP 70/30) or HAP/TCP 70/30 mixed with five percent strontium. Three months later animals were euthanazed, squared samples were cut out from the femur's body and later tested on a bend. The results of the research revealed that the femurs become more stringent after filling off defects in the greater trochanter zone with HAP/TCP 70/30 or HAP/TCP 70/30 mixed with five percent strontium. The ultimate pain for the cartilage component in the control groups was less, than in the test group. The flexure modulus of flexibility of a cartilage component in the test group was statistically reliable less, than the value of the flexure modulus of flexibility of the cartilage component in the control groups. Therefore, local usage of calcium - phosphatic bioceramic component over the greater trochanter part increases biomechanical characteristics of the cartilage component in the femur of animals.
\end{abstract}

Keywords: Biomechanical, Calcium phosphate, Osteoporosis, Rabbit, Strontium

\section{Introduction}

All types of biocomponent that is implanted into a living body elicits a distinct area reaction. This process depends on the effects of chemical, physic and physic-chemical properties of the component [1]. Biphasic calcium phosphate as cartilage grafting substitute is successfully used for filling of cartilage defects and local recovery of osteoporotic cartilage [2]. Strontium releases calcium phosphate of the cartilage cements which are prospective components for the clinical reconstruction of osteoporosis-related cartilage defects because it makes stimulation of cartilage formation as well as limits osteoclastic cartilage resorption [3, 4]. Our research objective was to define whether local use bioceramic component of Calcium phosphate in the femur greater trochanter area influences on the biomechanical attributes of the femur at animals with control osteoporosis.

\section{Component and Methods}

Control osteoporosis was activated in 19 eight month female rabbits by ovariectomy and following injections of methylpred- nisolone $1 \mathrm{mg} / \mathrm{kg}$ daily for 6 weeks. The test group consists of 4 female rabbits. On 15 animals defects in the greater trochanter zone were created, 8 of which have been stuffed with fragments of a hydroxyapatite and tricalcium phosphate (HAP/TCP 70/30); 7 defects have filled with HAP/TCP 70/30 mixed with five percent strontium. Three months later animals were euthanazed and squared samples (15 mm length) were cut out from the femur's body. Samples were smoothed.

Test of samples on a bend has been carried out at the Zwick/Roell stand (BT1-FR2.5TN.D14, Germany). The stand has been used together with the software testXpert 2.5 (Germany) for management of the equipment and data processing (Fig. $1)$.

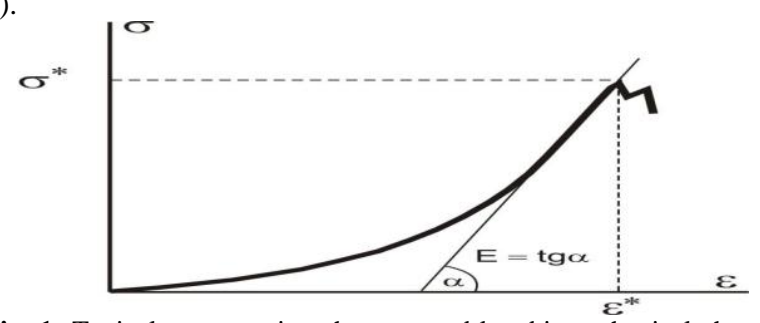

Fig. 1: Typical stress-strain coherence and key biomechanical characteristics: $\sigma^{*}$ - ultimate stress, $\varepsilon^{*}$ - ultimate strain and tangent $\alpha$-flexure modulus. 
In order to investigate the properties of a biomechanical bone component a three-point bending test was used [5]. Distances between the bending supports of the samples were $10 \mathrm{~mm}$. Width, length and thickness of samples were measured using a digital caliper; the accuracy of measurement was $\pm 0.01 \mathrm{~mm}$. Thickness of the samples was $0.62 \pm 0.01$; width: $3.2 \pm 0.01$. All biomechanical characteristics were introduced as medians (Md) with interquartile range (IQR). When the two groups were compared, the data was analyzed with the test of MannWhitney U. Two values are considered to be statistically significantly different if the confidence level of the difference between them is greater than $95 \%(\mathrm{p}<0.05)$.

\section{Results and Discussion}

Results of research have shown that ultimate stress of samples at the 3-point bending test for test group statistically did not differ from breaking points for samples from the experimental groups (Fig. 2).

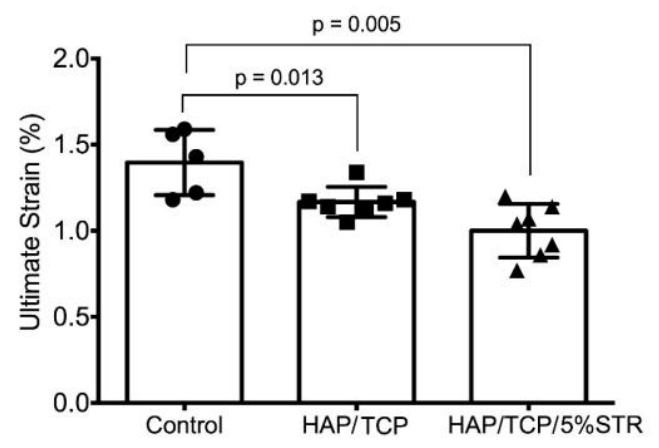

Fig. 2: Cross-sectional ultimate stress of the samples

Ultimate stress of samples in test group (Md $=176.7 \mathrm{MPa}(216$ - 154)) statistically reliable did not differ from the 2 nd group samples $(\mathrm{Md}=194.6 \mathrm{Mpa}(207.2-169.9))$, and the 3rd group samples $(\mathrm{Md}=193.7 \mathrm{MPa}(208.1-176.1))$, respectively. Ultimate stress of samples in the second and third group did not differ.

Investigation has shown that the ultimate strain for samples from test group is much higher, than for samples from two other groups (Fig. 3).

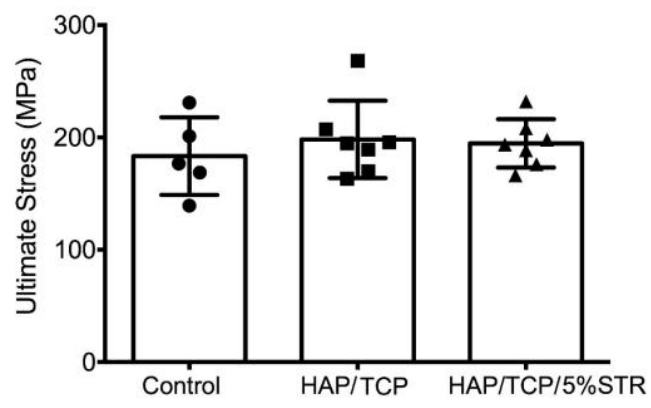

Fig. 3: Cross-sectional ultimate strain of the samples

For samples of test group the ultimate strain was $1.43 \%$ (1.571.20 ), that is significantly higher, than for samples of the 2nd group $(\mathrm{Md}=1.16 \%(1.18-1.13)), \mathrm{p}=0.013$ and for samples of the 3rd group $(\mathrm{Md}=1.04 \%(1.14-0.86)), \mathrm{p}=0.005$, respectively. Between the ultimate strain of samples from the 2 nd and 3 rd groups statistically reliable differences $(p=0.058)$ did not revealed.

The flexure modulus of flexibility characterizes rigidity of component, and if the importance of the flexure modulus of flexibility is higher, the component is more rigid. The analysis of the received results has shown that the flexure modulus of flexibility of a cartilage component in the test group was 16.31
GPa (18.66 - 12.15), that is statistically reliable less ( $p=0.03)$, than the importance of the flexure modulus of flexibility of the cartilage component in the second group $(\mathrm{Md}=19.87 \mathrm{GPa}$ $(21.10-17.94))$ and in the third group $(\mathrm{Md}=21.88 \mathrm{GPa}(27.53$ $-21.09)), p=0.005$, respectively. Flexure modulus of samples in the 2nd and 3rd group statistically did not differ $(\mathrm{p}=0.053)$ (Fig. 4).

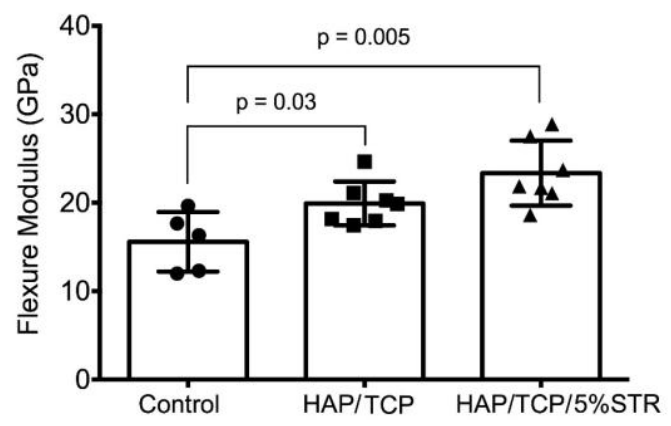

Fig. 4: Cross-sectional flexure modulus of the samples

\section{Conclusion}

Results of research show, that the femur body in rabbit becomes more stringent after filling off defects in the greater trochanter area with fragments of a hydroxyapatite and tricalcium phosphate (HAP/TCP 70/30) or HAP/TCP 70/30 mixed with five percent strontium. The eventual injury in the cartilage component in the experimental groups is fewer, than in the test group. The flexure modulus of flexibility of a cartilage component in the test group was statistically reliable less, than the importance of the flexure modulus of flexibility of the cartilage component in the experimental groups. Therefore, local usage of the calcium-phosphatic bioceramic substance over the greater trochanter area improves biomechanical characteristics of a cartilage component in the femur of animals.

\section{Acknowledgments}

Study was supported by National Research Programme No. 2014.10-4/VPP-3/21 "Multifunctional Components and composites, photonics and nanotechnology". Project No. 4 "Nanocomponents and nanotechnologies for medical applications".

\section{References}

[1]. Chen T, Chen M, Yau X, Long W, Chen H, Li L, A new threepoint bendind test for cartilage biomechanical properties of rat's tibia, Sheng Wu Yi Xue Gong Cheng XueZaZhi 2 (2008), 341345.

[2]. Dorozhkin SV, Biphasic, triphasic and multiphasic calcium orthophosphates, Acta biomater. 8 (2012), 963-977.

[3]. Lode A, Heiss C, Knapp G, Thomas J, Nies B, Gelinsky M Schumacher M, Strontium-modified premixed calcium phophate cements for the therapy of osteoporotic cartilage defects, Acta Biomater.17 (2017), 30664-30665.

[4]. Lozano D, Trejo CG, Gomez-Barrena E, Manzano M, Doadrio JC, Salinas AJ, Vallet-Regi M, Garcia-Honduvilla N, Esbrit P, Bujan J, Osteostatin-loaded onto mesoporous ceramics improves the early phase of cartilage reconstruction in a rabbit osteopenia model, ActaBiomater. 8(6) (2012), 2317-2323.

[5]. Slutskii L, Vetra J, Letter to the editor: Biocompatibility and reactogenicity of components: a semantic and logical analysis of definitions of their practical significance, Cells and Components 6(1-3) (1996), 137-142. 